Peer Reviewed Paper openaccess

\title{
Development of a hyperspectral imaging technique with internal scene scan for analysing the chemistry of food degradation
}

\author{
Guo Hsuan Chen ${ }^{\mathrm{a}}$ and Jung Y. Huang ${ }^{\mathrm{b}, *}$ \\ The T.K.B. Research Center of Photonics, Chiao Tung University, Hsinchu 300, Taiwan. ORCID: https://orcid.org/0000-0001-7916-8292 \\ bThe T.K.B. Research Center of Photonics, Chiao Tung University, Hsinchu 300, Taiwan. E-mail: jyhuang@faculty.nctu.edu.tw. \\ ORCID: https://orcid.org/0000-0002-4189-3779
}

\begin{abstract}
Hyperspectral imaging (HSI) can provide valuable information about the spatial distribution of ingredients in an object, therefore the technique has been widely adopted in numerous applications, ranging from remote sensing and land planning, food quality control, to biomedical applications. However, HSI instruments are expensive, which has limited the technique to some high-end applications. In this study, we developed a cost-effective HSI technique with an internal scene-scan mechanism, which enables rapid acquisitions of a scene without moving the instrument or the tested object. The apparatus was characterised, revealing an imaging resolution of $0.4 \mathrm{~mm}$ in a field of view (FoV) of $10 \mathrm{~cm}$ and a spectral resolution of $1.3 \mathrm{~nm}$ in the $400-800 \mathrm{~nm}$ visible light region. We succeeded in applying our apparatus to analyse the oxidation processes of apple and meat, which demonstrated our design and relevant data analysis to be of high value to visualise chemistry related to food quality and safety.
\end{abstract}

Keywords: hyperspectral imaging, diffuse reflectance, visible light, food quality control, telecentric, principal component analysis, L2 norm, spectral angle, $k$-mean clustering

\section{Introduction}

The interaction of light with biological tissue is an important problem in the development of diagnostic methods with light. ${ }^{1,2}$ As photons propagate through biological tissue, they may encounter absorption and multiple scattering. In the wavelength window of 600-1300 nm, where most biological tissues have sufficiently weak absorbances, ${ }^{3}$ optical scattering becomes the major player that makes light propagation diffuse. In that case, we can measure the diffuse reflectance signal to monitor local changes in scattering and absorption properties of tissue and, thereby, non-invasively, probe the structures, metabolism and microenvironment in tissue.
Hyperspectral imaging (HSI) had been developed to capture the reflected, fluorescent and transmitted light from tissue to make direct identification of different constituents and their spatial distribution. ${ }^{2}$ The resulting data hypercube I $(x, y, \lambda)$ comprises two spatial dimensions and one spectral dimension. ${ }^{4}$ Typical data acquisition with HSI involves the use of an array of detectors multiplexed in two dimensions and, therefore, requires multiple measurements to complete a data hypercube. The multiple measurements are often implemented as either position scanning or wavelength scanning. ${ }^{5}$ Position scanning HSI systems acquire a 2D data of one spatial

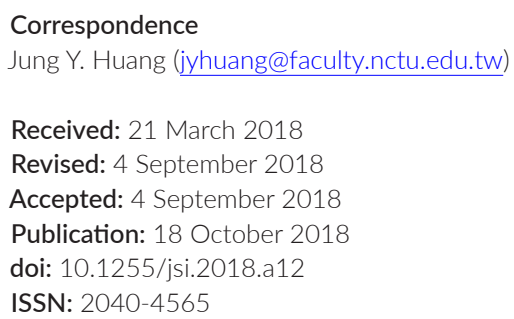

\section{Citation}

G.H. Chen and J.Y. Huang, "Development of a hyperspectral imaging technique with internal scene scan for analysing the chemistry of food degradation", J. Spectral Imaging 7, a12 (2018). https://doi.org/10.1255/ jsi.2018.a12

(C) 2018 The Authors

This licence permits you to use, share, copy and redistribute the paper in any medium or any format provided that a full citation to the original paper in this journal is given. 
dimension and the spectral dimension, and scan across the other spatial dimension. Wavelength scanning types multiplex the two spatial dimensions and scan across the spectral dimension.

Enabling fast hyperspectral imaging with highthroughput can open doors to new studies of complex systems where multiple constituents need to be resolved. A variety of snapshot techniques have been developed to improve the speed of operation and increase the light throughput. 6 Typically, these snapshot techniques can be realised based on a spatial-spectral modulation scheme, which either generates a coded light pattern on an object ${ }^{7}$ or inserts a spectral modulation module in an imaging device. ${ }^{6,8}$ For example, such coded light patterns for object illumination can be generated by a digital micromirror device. ${ }^{7}$ Pixelated filters (either interference type ${ }^{9}$ or diffraction type ${ }^{10}$ ), ordinary refractive prisms ${ }^{11}$ or Michelson interferometers ${ }^{4,7}$ are suited to equip an imaging device with spectral resolving power. However, most of the proposed snapshot techniques require a computational image formation model to recover images at discrete wavelengths. Many HSI applications require high constituent discrimination, which may be better served with continuous spectral profiles with rich spectral characteristics.

Due to the wide demand for high spectral and spatial resolution in $\mathrm{HSI}$ apparatus, it is highly desirable to design and implement a cost-effective HSI system. Line-scan HSI systems, mainly used for rapid surface inspection of an object, use line light sources to build a complete hypercube $I(x, y, \lambda)$ line by line by translating the object along one spatial direction. ${ }^{12}$ In this paper we present a HSI system with an internal scene scan, which can rapidly acquire an entire scene without moving the instrument or object. Telecentric optics ${ }^{13}$ were implemented to yield a long focal depth. The resulting apparatus was characterised and yielded an imaging resolution of $0.4 \mathrm{~mm}$ in a $10 \times 10 \mathrm{~cm}$ field of view (FoV) and a spectral resolution of $1.3 \mathrm{~nm}$ in the $400-800 \mathrm{~nm}$ visible light region. Our HSI instrument design can be built at a total cost of $<$ US \$10K (see Table 1).

Although reagent assay provides a more accurate and objective evaluation of food status, it requires special reagents and protocols to yield an accurate result. So, in the past decades, consumers routinely relied on colour, smell and appearance to select or reject food products. ${ }^{14}$ Developing a quality evaluation method to assess the food quality parameters and to ensure its authentication would bring economic benefits to the food industry by increasing consumer confidence in the quality of food products.

The main attributes of meat have been established and include the water-holding capacity, colour, fat content, $\mathrm{pH}$ and oxidative stability. HSI has the potential to

Table 1. Component list of the HSI apparatus.

\begin{tabular}{|c|c|c|}
\hline Components & Spec & Unit price (US\$) \\
\hline T1 & $\begin{array}{l}\text { Achromatic doublet lens: dia. }=2.5 \mathrm{~cm}, \mathrm{FL}=20 \mathrm{~cm}, \mathrm{MgF}_{2} \text {-coated; } \\
\text { Edmund 32-917 }\end{array}$ & 131 \\
\hline SM & $\begin{array}{l}\text { Single-axis scanning system: max. scan angle }=20^{\circ} \text {, step response }= \\
400 \mu \mathrm{s} \text {; Thorlabs GVS211 }\end{array}$ & 3800 \\
\hline TF & $\begin{array}{l}\text { F-Theta lens: } \mathrm{WD}=10 \mathrm{~cm}, \mathrm{EFL}=10 \mathrm{~cm}, \text { scan angle }=12.5^{\circ} \\
\text { Edmund } 64-426\end{array}$ & 1633 \\
\hline T2 & $\begin{array}{l}\text { Achromatic doublet lens: dia. }=3 \mathrm{~cm}, \mathrm{FL}=5 \mathrm{~cm}, \mathrm{MgF}_{2} \text {-coated; } \\
\text { Edmund } 32-496\end{array}$ & 146 \\
\hline S & Adjustable slit: $3 \mathrm{~cm}$ cage system; Thorlabs VA100C & 329 \\
\hline LG-1 & Aspheric lens: dia. $=2.5 \mathrm{~cm}, \mathrm{FL}=2 \mathrm{~cm}, \mathrm{NA}=0.54 ;$ Thorlabs AL2520 & 276 \\
\hline LG-2 & $\begin{array}{l}\text { Transmission grating: } 600 \text { grooves } \mathrm{mm}^{-1} \text {, Groove angle }=28.7^{\circ} \text {; } \\
\text { Thorlabs GT13-06V }\end{array}$ & 80 \\
\hline IL & Aspheric achromatic lens: dia. $=2.5 \mathrm{~cm}, \mathrm{EFL}=3 \mathrm{~cm}$; Edmund 49-662 & 170 \\
\hline Camera & $\begin{array}{l}2048 \times 2048, \text { active area }=1.1 \times 1.1 \mathrm{~cm}, \text { pixel size }=5.5 \mu \mathrm{m} \\
\text { Ximea MQ042MG-CM }\end{array}$ & 3000 \\
\hline Total & & 9565 \\
\hline
\end{tabular}


objectively assess meat quality by providing valuable information about ingredient compositions and spatial distribution. ${ }^{12,15-18}$ For example, diffuse reflectance signals at $459,618,655,685,755$ and $953 \mathrm{~nm}$ were found to correlate strongly with the drip-loss of pork. High correlations were also found at 494, 571, 637, 669, 703 and $978 \mathrm{~nm}$ for $\mathrm{pH}$, at 434, 494, 561, 637, 669 and $703 \mathrm{~nm}$ for colour, and at $772 \mathrm{~nm}$ for beef tenderness. ${ }^{15}$ Similarly, in the NIR region, some wavelengths at 1074, 1091, 1142, 1176, 1219, 1365, 1395, 1408 and $1462 \mathrm{~nm}$ corresponding to fat, protein and water absorptions were identified. ${ }^{15}$ Light scattering also can be a useful measure. Indeed, it was revealed that the changes in scattering profiles from $1000 \mathrm{~nm}$ to $1900 \mathrm{~nm}$ correlate well with beef tenderness. ${ }^{15}$

Considering the nutritional value of fruit products, apples are major contributors of phenolics and flavonoids in our diet that can provide antioxidant protective effects in the body. A Cornell group led by R. Liu discovered that the total extractable phenolic content ranges from $110 \mathrm{mg}$ per $100 \mathrm{~g}$ to $357 \mathrm{mg}$ per $100 \mathrm{~g}$ of fresh apple and exhibits high antioxidant activity. ${ }^{19}$ Polyphenol oxidase (PPO), rich in the flesh portion of an apple, can catalyse browning reactions, ${ }^{20}$ which involves the oxidation of phenolic compounds to quinones and eventual polymerisation to melanin pigments. These reactions proceeding in many foods of plant origin cause deterioration and loss of food quality.

We verified the functionality of our HSI design and efficacy of the data analysis procedure by examining the oxidation processes of apple and meat. The results demonstrated that our HSI design can be of high value to analyse the degradation chemistry of fruit and meat for food quality control.

\section{Instrumental design}

As a tested object moves behind an imaging lens, the image size varies with the object location when the image is taken. From an application point of view, the optical metrology apparatus should yield a sharp image with a constant dimension as the object position changes. Our HSI design addresses this concern with the following three design objectives. First, the HSI instrument should possess good imaging quality with a long focal depth, while maintaining a constant magnification. Second, to yield maximum material discrimination the HSI design should also provide a continuous spectral profile instead of a few discrete bands. Third, we would like to capture the entire scene with an internal scene-scan scheme without moving either apparatus or object.

Figure 1(a) depicts the principle of our design. In brief, an achromatic lens $(T 1, f=20 \mathrm{~cm})$ collects the light from an object and transforms it to a collimated beam at the position of the scanning mirror (SM). Telecentric optics are formed by pairing $T 1$ with $T 2(f=5 \mathrm{~cm}) .{ }^{13}$ The small SM also serves as a light-limiting aperture, which prevents off-axis light from reaching T2.

To realise an internal scene-scan mechanism, we inserted a telecentric $f-\theta$ module (TF) between SM and T2. Although the telecentric lens can focus incident light on one plane, those beams can arrive on the plane at different angles [see the bottom schematic of Figure $1(b)]$, whereas a telecentric $f-\theta$ lens can make those incident beams perpendicular to the focal plane [the top schematic of Figure 1(b)]. By properly choosing T1 and $\mathrm{T} 2$, we were able to shrink an entire $10 \times 10 \mathrm{~cm}$ FoV and bring it to a slit (S) perpendicularly [see ray-tracing result of Figure 1(c)], which yields a laterally shifted image with a shift distance linearly proportional to the angle of SM. Thereby, by combining SM and S, we can sequentially cut a 2D scene into lines.

We designed a two-lens spectrograph to generate continuous spectral profiles to yield high material discrimination. We fabricated a LG by adhesively bonding an aspheric lens $(f=2.5 \mathrm{~cm})$ to a transmission grating $\left(600\right.$ grooves $\mathrm{mm}^{-1}$ ) and positioned the device directly behind the slit to disperse the line-shaped light. The spectrally dispersed light was then imaged with an achromatic lens (IL, $f=3 \mathrm{~cm}$ ) and captured by a CMOS camera. The camera has an image format of $2048 \times 2048$ with a pixel size of $5.5 \mu \mathrm{m}$.

\section{System characterisation Spectral characterisation}

We built the HSI apparatus and characterised its imaging spectroscopic functionality. For the convenience of readers, all components of this setup are summarised in Table 1 with relevant specifications and unit prices. In the first step, we calibrated the wavelength at each pixel of our camera using a cascaded monochromator and tungstenhalogen lamp. The light source is wavelength tuneable from $400 \mathrm{~nm}$ to $800 \mathrm{~nm}$ with a bandwidth of $10 \mathrm{~nm}$. After 


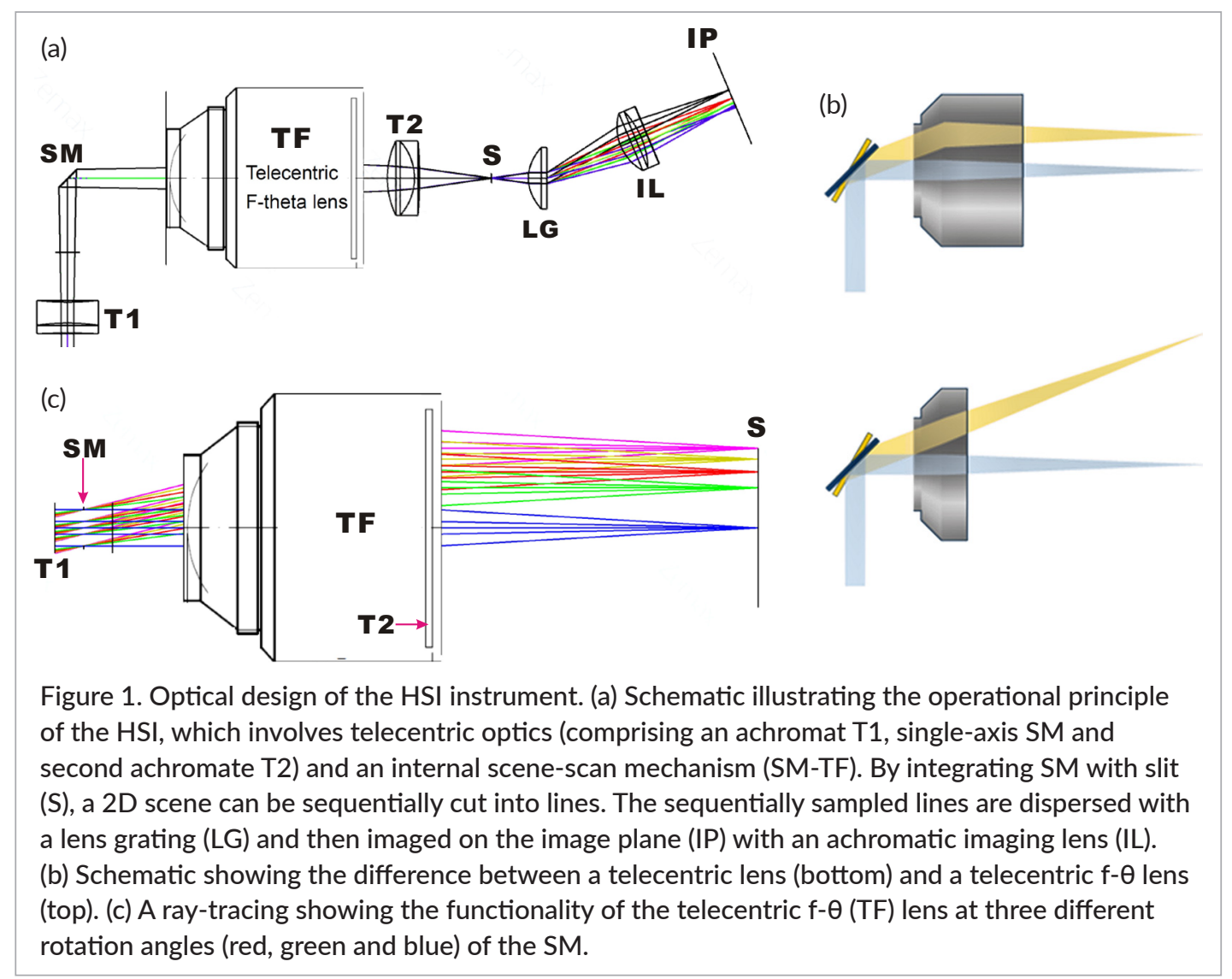

the calibration, we measured the spectral sensitivity $P(\lambda)$ of the camera. By combining these two calibration results, we moved on to measure the broadband spectrum of the tungsten-halogen lamp, $S(\lambda)$, by rotating the grating of the monochromator to zero-order diffraction. As shown in Figure 2(a), the spectrum measured by our HSI instrument (blue symbols) is similar to that of a commercial spectrograph (Ocean Optics USB4000, red line). To verify that our HSI instrument can also yield accurate spectra from a diffuse reflectance surface, we recorded the light of the tungsten-halogen lamp reflected from a surface with a Labsphere Spectraflect diffuse reflectance coating (D95). Note that the measured diffuse reflectance signal can be expressed as $I_{0}(\lambda)=S(\lambda) D_{95}(\lambda) P(\lambda)$. Figure 2(b) presents the result of $D_{95}(\lambda)=I_{0}(\lambda) /[S(\lambda) P(\lambda)]$, which reveals a fairly flat profile, agreeing well with the specification data published in the literature. ${ }^{21}$ The flat spectral profile of D95 suggests this white surface is a convenient standard to be used to determine a unknown diffuse reflectance spectrum. The diffuse reflectance image from an object under test can be expressed as $I(x, y, \lambda)=S(\lambda) O(x, y, \lambda) P(\lambda)$ with $O(x, y, \lambda)$ being the diffuse reflectance of the object, which can be determined with $O(x, y, \lambda)=D_{95} \cdot /(x, y, \lambda) / I_{0}(\lambda)$.
Our HSI instrument also yields good spectral resolution in the visible light region. The measured spectral profile of a He-Ne laser at $632.8 \mathrm{~nm}$ is shown in Figure 2(c). Fitting the spectral profile to a Gaussian function results in a full width of 4.5 pixels at half maximum, indicating a spectral resolution of $1.3 \mathrm{~nm}$ for visible light.

\section{Spatial characterisation}

We designed a grid pattern to test the edge resolution of our $\mathrm{HSI}$ instrument. By positioning the camera and the $\mathrm{IL}$ at the zero-order diffraction angle of the $\mathrm{LG}$, the observed image without slit $\mathrm{S}$ is shown in the left photo in the inset of Figure 3. The corresponding edge profile is depicted with open squares. The right photo was taken through a 0.1-mm wide slit whilst scanning the SM. The resulting edge profile is displayed with red-filled circles. We can fit the edge profiles to an error function, which yields a spatial resolution of $0.6 \mathrm{~mm}$ for direct imaging and $0.4 \mathrm{~mm}$ for line-scan imaging.

We simulated the image formation with a ray-tracing technique. Placing a point-like source on the object surface, produced a 0.4-mm spot on the slit. Next, we placed pointlike sources with diameters of $0.1 \mathrm{~mm}$ and $0.4 \mathrm{~mm}$ on the 

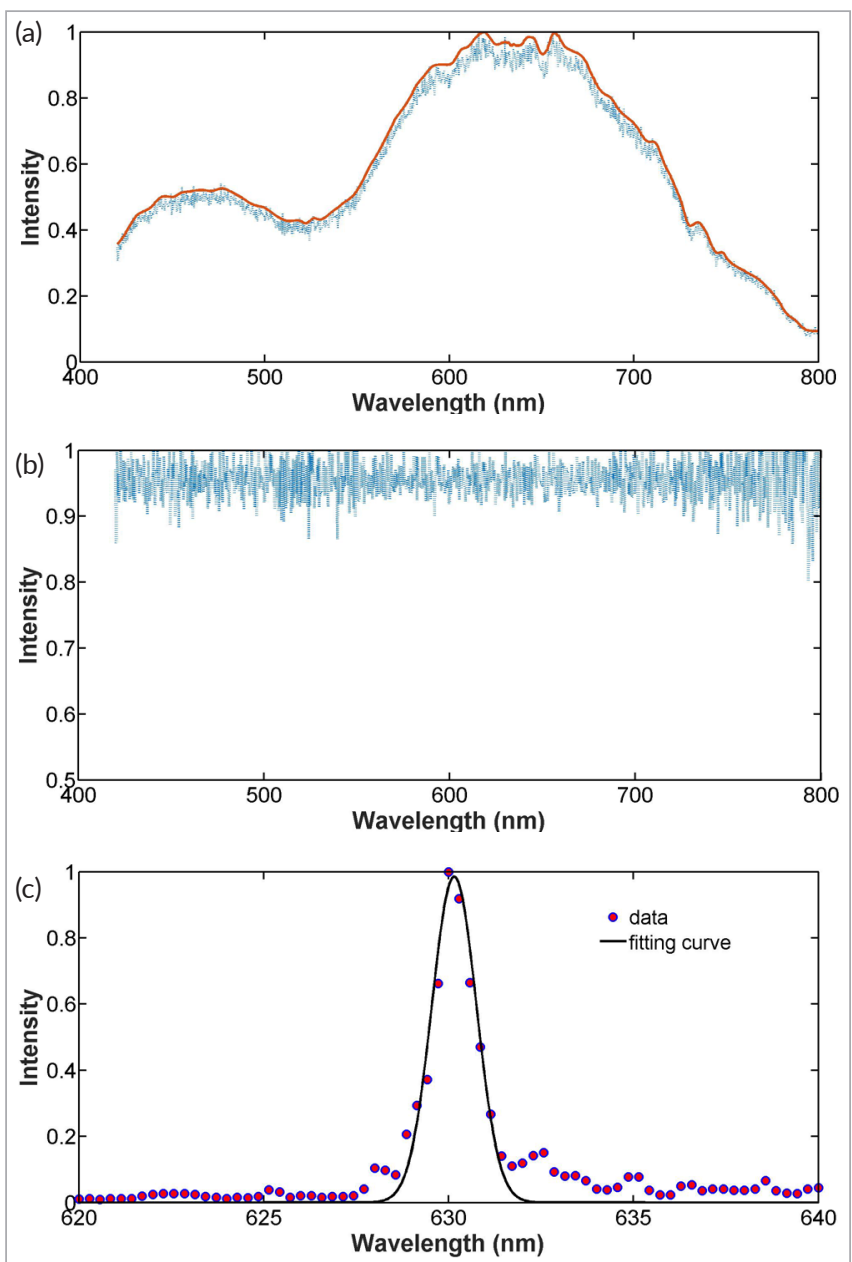

Figure 2. Performance of the HSI instrument. (a) Broadband spectrum of tungsten-halogen lamp $S(\lambda)$ measured by the instrument (blue symbols) and a commercial spectrograph (red line). (b) Reflectance spectrum from a diffuse white surface (D95) measured by the imager. (c) The He-Ne laser spectrum measured by the HSI instrument yielded a spectral resolution of $1.3 \mathrm{~nm}$.

slit and simulated the image formed by the imaging spectrograph. At the IP, both the $0.1 \mathrm{~mm}$ and $0.4 \mathrm{~mm}$ sources produce an image spot with a diameter of $0.57 \mathrm{~mm}$ and $0.44 \mathrm{~mm}$ with direct and line-scan imaging, respectively, suggesting that the $0.1 \mathrm{~mm}$ diameter light spot behaves like a point source for the imaging spectrograph. Thus, we can conclude that a $0.1-\mathrm{mm}$ wide slit effectively matches to the imaging spectrograph and is optimal for our HSI instrument design. The line-scan imaging with a slit has better resolution than that with direct imaging.

\section{Data processing and analysis}

Typical data processing and analysis for HSI can be summarised as image preprocessing, ${ }^{22}$ feature extraction, ${ }^{23,24}$ and classification. ${ }^{25,26}$ The detailed description of each algorithm involved is out of the scope of this paper. Fortunately, there are excellent review articles or tutorials on these subjects in literature. ${ }^{27-29}$ Below, we will focus on HSI applications in food safety assessment and use these examples to elaborate relevant data processing and analysis methods used in this study. In brief, the measured $\mathrm{HSI}$ data I( $x, y, \lambda)$ are first preprocessed with background removal and then normalised by the reflectance spectrum from D95, which yields a diffuse reflectance spectra of object $O(x, y, \lambda)$. The reflectance spectra were then denoised along the spectral dimension with a minimum noise fraction algorithm. ${ }^{30}$

Principal component analysis (PCA) ${ }^{31}$ is useful for $\mathrm{HSI}$ data analysis. We performed PCA and k-mean clustering on the preprocessed HSI hypercubes by using SIproc, ${ }^{32}$ which is an open-source biomedical data processing platform and available from the website at http://stim.ee.uh. edu/resources/software/siproc/. This software tool can alleviate memory limitations and allows users to process hyperspectral images on a computer with limited system memory.

\section{Applications}

\section{Observing the browning reaction of apple}

We applied our HSI instrument to analyse PPO-catalysed browning of an Empire apple, which is a cross between Red Delicious and Mclntosh developed by the New York State Agricultural Experiment Station. We took HSI images of a fresh-cut apple every 30 min for a total duration of $2.5 \mathrm{~h}$. The measured $\mathrm{O}(\mathrm{x}, \mathrm{y}, \lambda)$ spectra are displayed in Figure 4. We observed a peak at $620 \mathrm{~nm}$, which can be attributed to the charge-transfer transition of $\mathrm{O}_{2} \rightarrow \mathrm{Cu}(\mathrm{II})$ in PPO. ${ }^{33}$ The peak weakens with time, suggesting that the PPO in the flesh portion was depleted with time.

The time series HSI data comprise multiple hypercubes, each presenting the sample at a different time point. Consider each spectrum in a hypercube as an $N$-dimensional vector $\vec{U}=\left(u_{1}, u_{2}, \ldots, u_{N}\right)$. To quantify the difference between two spectra, we invoked the $L 2$ norm difference defined by

$$
\Delta_{U V}=\sqrt{\sum_{i=1}^{N}\left|u_{i}-v_{i}\right|^{2}}, 34,35
$$

where $u_{i}$ and $v_{i}$ denote the corresponding intensities at pixel $i$ along the spectral dimension. As displayed in Figure $5(\mathrm{~b})$, the histogram of $\triangle_{U V}$ over an image varies 


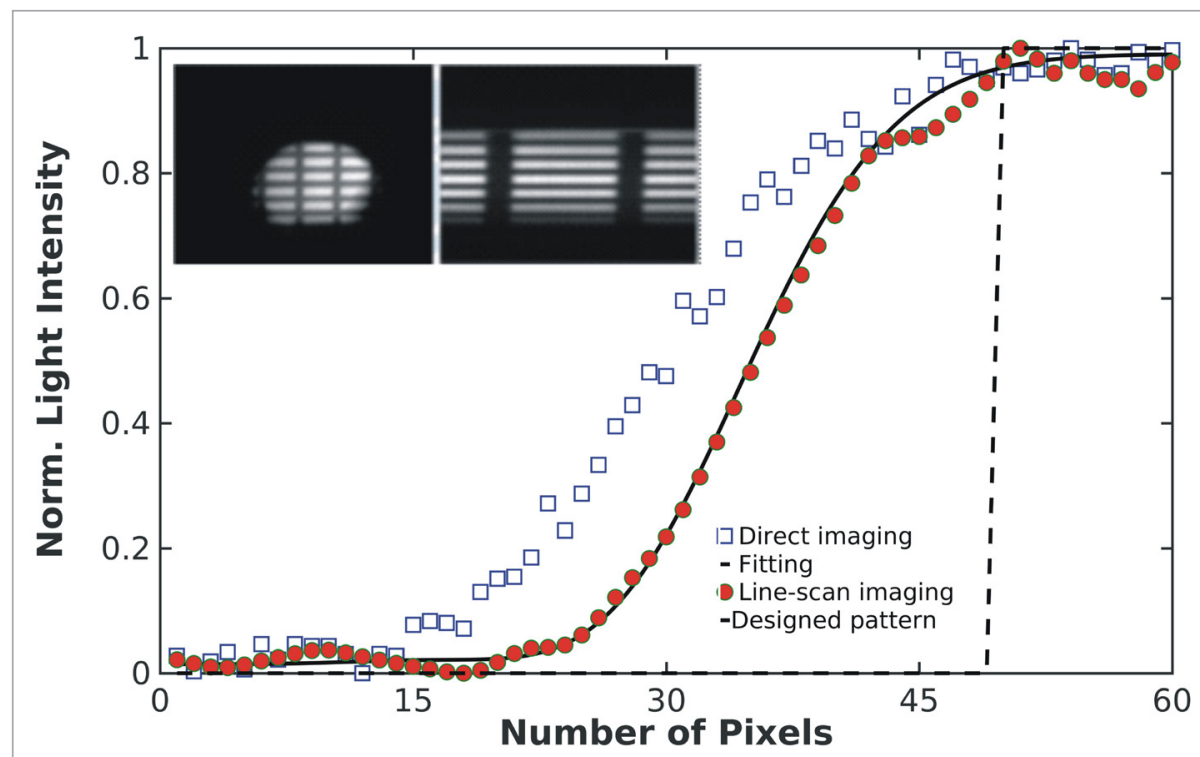

Figure 3. Spatial resolution of the HSI instrument. Left photo of the inset: image of the grid pattern acquired by direct imaging, right photo: line scanning image using a $0.1-\mathrm{mm}$ wide slit. The direct imaging profile is shown by open squares and the linescanning image is shown by filled circles with the solid line being the fitting curve. Each pixel has a dimension of $5.5 \mu \mathrm{m}$.

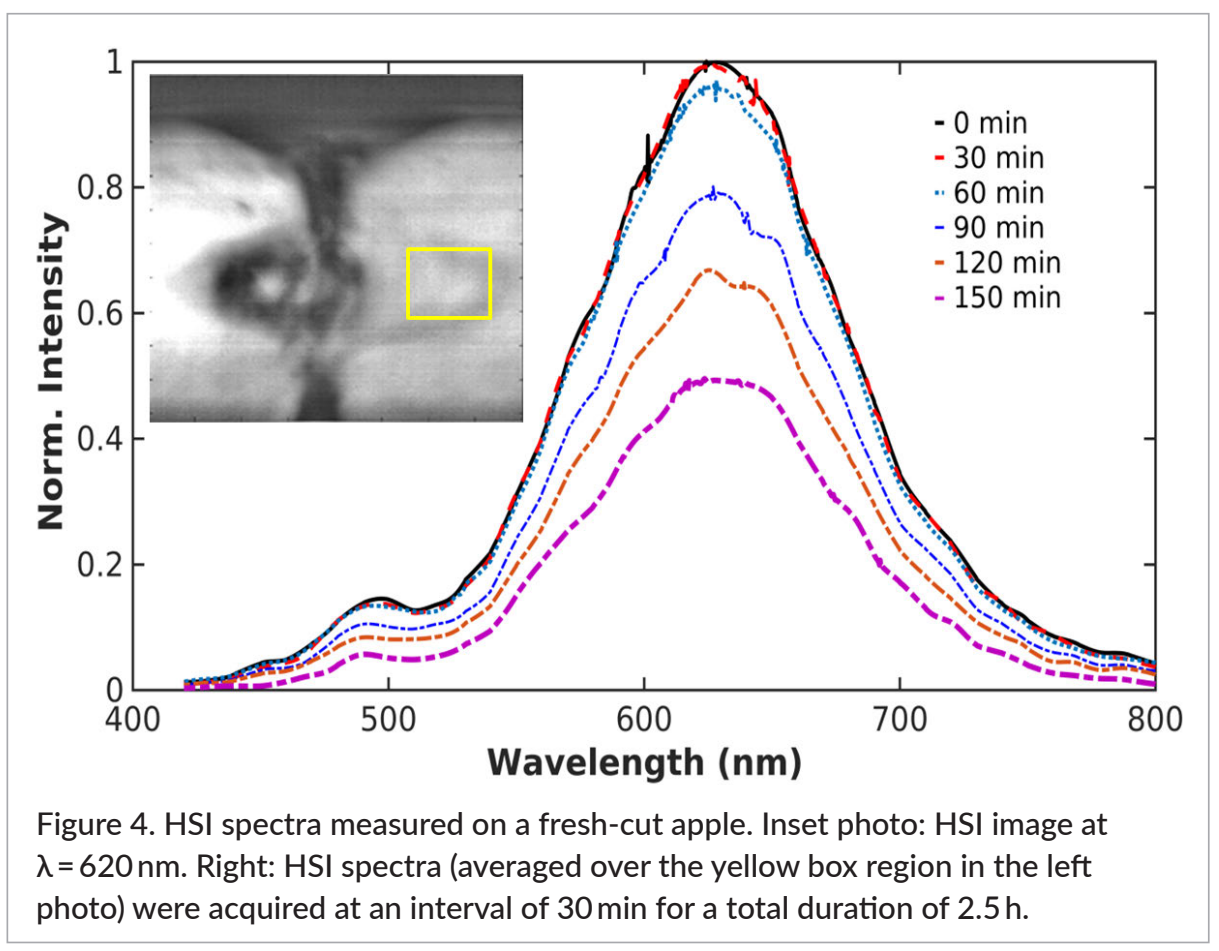

with time, indicating temporal changes of the degree of oxidation. The spatial distribution of $\triangle_{U V}$ at a different time point is shown in Figure 5(a), revealing that most of the oxidation occurs in the flesh or seed, where PPO is abundant.
We performed PCA on the entire hypercube at $t=150$ min using the SIproc software tool. ${ }^{32}$ After the PCA analysis, we applied a k-mean clustering technique to separate pixels into four clusters centred at the first four PCA components using $\triangle_{U V}$ as a simi- 


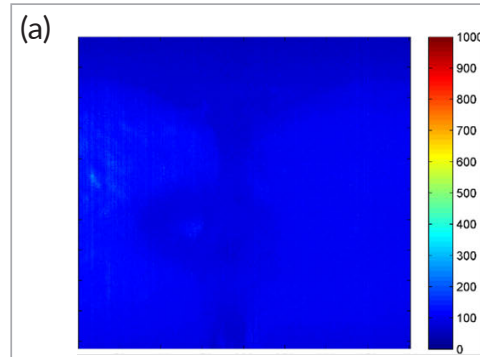

(i)

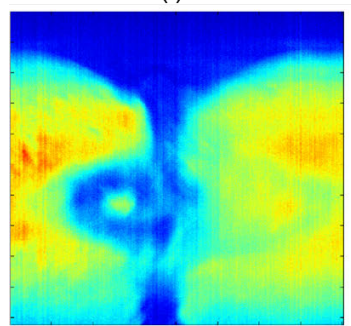

(iv)

(b)

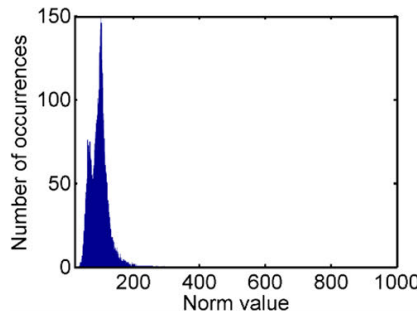

(i)

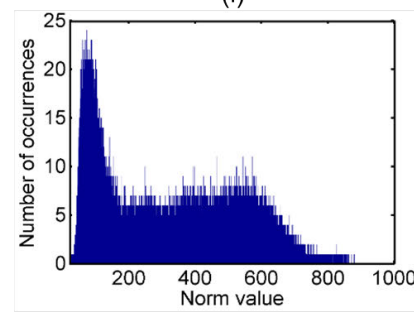

(iv)

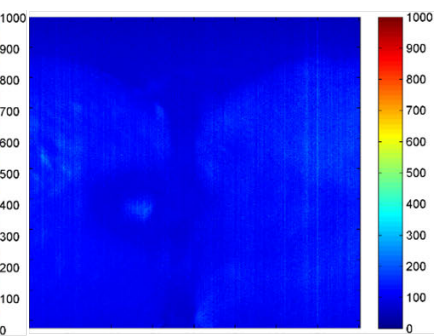

(ii)

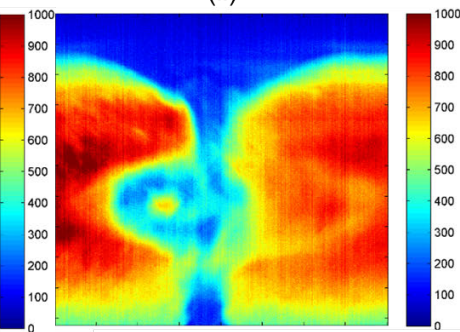

(v)

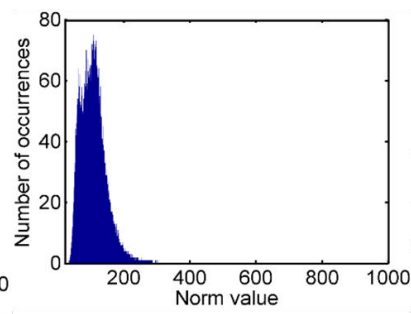

(ii)

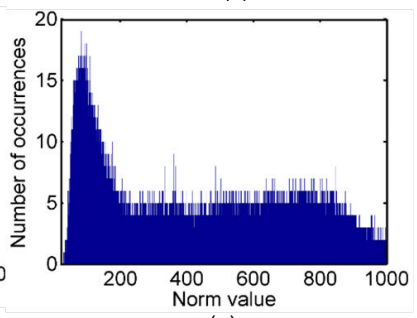

(v)

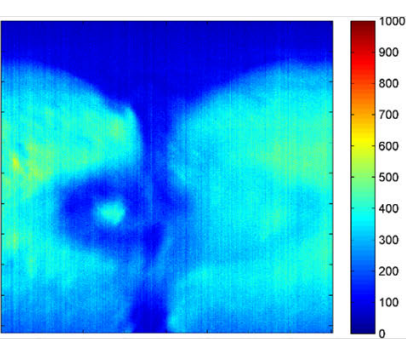

(iii)

Figure 5. HSI measurement on an apple. (a) Time series $\Delta_{U V}$ images. (i)-(v): $30-150 \mathrm{~min}$ (b) The corresponding histograms of $\Delta_{U V}$.

larity measure. The result is presented on the right of Figure 6(a). Four regions with peel, peel + flesh, flesh and nucleus were identified with different degrees of oxidation. Flesh is the region with the highest degree of oxidation (red), whereas the lowest oxidation occurs in peel and in some parts of nucleus (blue). The degree of oxidation decreases from flesh towards peel and nucleus.

This result from HSI is reasonable, considering that phenolic and flavonoid provide a strong antioxidant effect. The clustering of HSI data also correlates with the phenolic and flavonoid content of apples, ${ }^{19}$ suggesting that the total phenolic and flavonoid contents are highest in the peels, followed by the flesh + peel and the flesh of apples. By setting the initial fraction of no oxidation class to be 100 , we can deduce relative percentages of four classes as a function of time. The result is presented in Figure $6(b)$. The relative percentages of the four clusters vary with the elapsed time and the effect of browning reaction is visible (see the light blue bars) at $90 \mathrm{~min}$ after the surface is exposed.

\section{Visualising the degradation chemistry of meat}

Myoglobin (Mb) is the primary red pigment in meat, which makes colour evaluation an essential part of meat selection and troubleshooting of processing problems. ${ }^{14-16}$ 

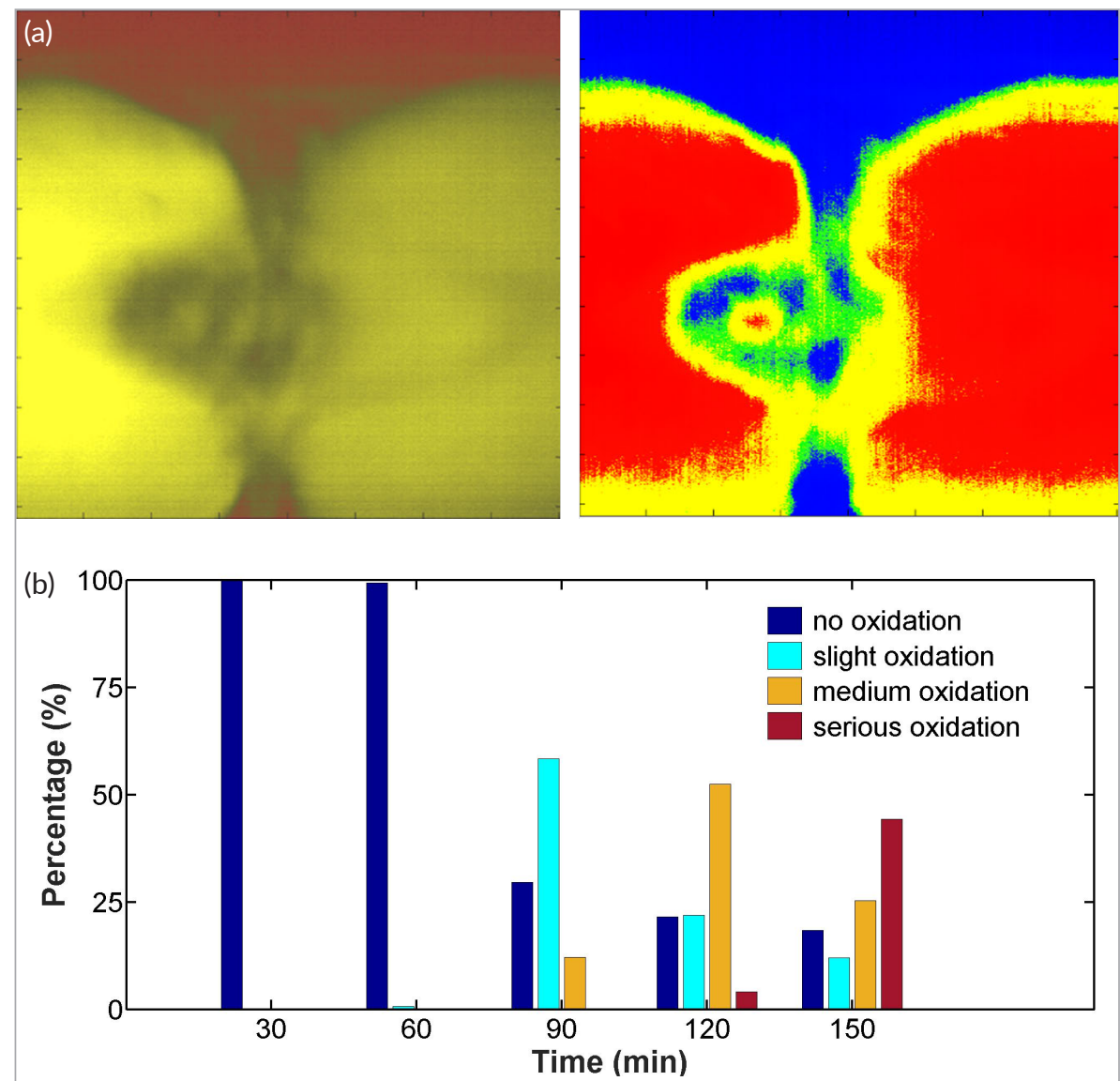

Figure 6. PCA result of HSI data. (a) Left photo: HSI image at $620 \mathrm{~nm}$. Right photo: pixel clustering result of $\mathrm{HSI}$ data at $t=150 \mathrm{~min}$. (b) Relative percentages of $\mathrm{HSI}$ classes versus the elapsed time.

Ligand present at one of the axial coordination sites of the haeme group of $\mathrm{Mb}$ changes the valence state of iron and determines the meat colour. It is known that the following four species of Mb can be formed: deoxymyoglobin (DeoxyMb), oxymyoglobin (OxyMb), carboxymyoglobin (COMb) and methyl myoglobin (MetMb). Post- mortem bacteria breeding causes irreversible changes of $\mathrm{Mb}$ from DeoxyMb and OxyMb to MetMb. From the point of view of food safety and assessment, it is important to assay the content of MetMb in meat. In this section, we will show how HSI can help in evaluating the degree of methylation of $\mathrm{Mb}$ in meat.

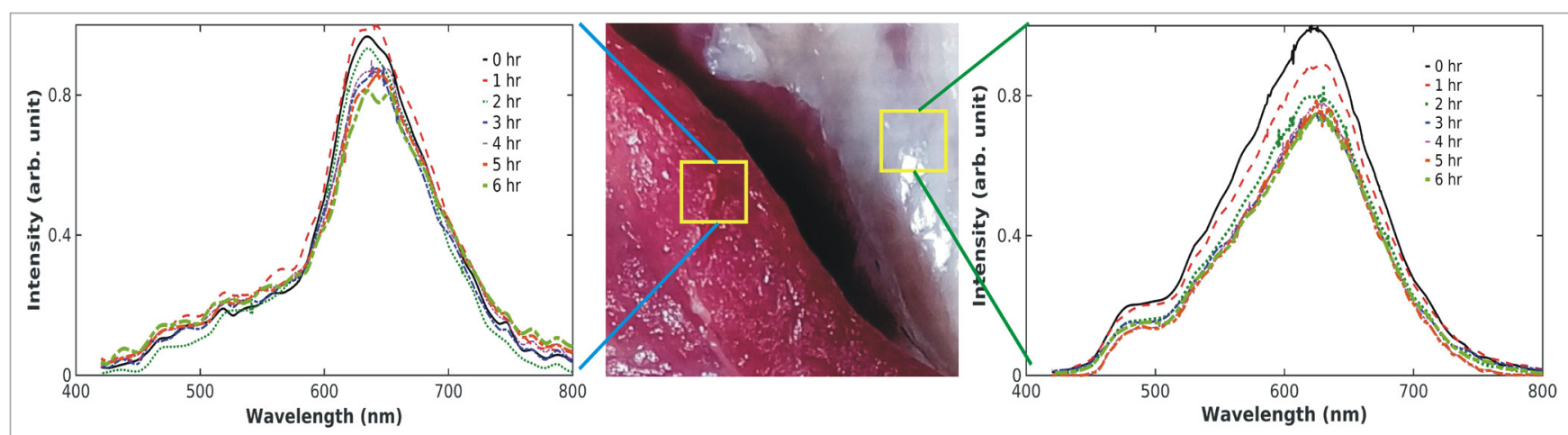

Figure 7. Digital RGB colour photo of pork. HSI spectra (averaged over the yellow boxes in the photo) measured on the red meat (left) and fat tissue (right) at an interval of $1 \mathrm{~h}$ for a duration of $6 \mathrm{~h}$. 
A digital RGB colour image taken on a piece of pork from a nearby abattoir one hour after being sacrificed is displayed in the centre of Figure 7. As revealed, the pork contains a combination of red meat and fat. We then collected the HSI data at an interval of $1 \mathrm{~h}$ for a duration of $6 \mathrm{~h}$. The resulting spectra from the fat (right) and red meat (left) region are also presented in Figure 7. Noticeable changes can be observed. The spectrum of fat tissue decreases with time without significant changes in the shape of its spectral profile, whereas complicated variation can be detected in the red meat region. The spectral intensity between $600 \mathrm{~nm}$ and $700 \mathrm{~nm}$ rises in the first hour and then decreases with time. The main peak at $620 \mathrm{~nm}$ splits into several subpeaks with peak wavelength shifting with time.

We performed PCA on pixel-wise spectra at $t=0$. After the PCA procedure, we applied a k-mean clustering technique to separate pixels into two clusters centred on the first two PCA components. For k-mean clustering, spectral angle was used to provide a similarity measure for spectral vectors $U$ and $V$, which is defined by

$$
A_{u V}=\cos ^{-1}\left[\sum_{i=1}^{N} u_{i} v_{i} /\left(\sqrt{\sum_{i=1}^{N}\left|u_{i}\right|^{2}} \sqrt{\sum_{i=1}^{N}\left|v_{i}\right|^{2}}\right)\right] \cdot 34,35
$$

The result is presented on the right of Figure 8 in red and blue. As shown, the first two PCA components can label the red meat (red) and fat (blue) correctly. ${ }^{15,17}$ The lowerleft region was attributed to fat, presumably the top layer is saturated with fat, and demonstrates that HSI has a better material discrimination power than digital RGB colour imaging shown on the left of Figure 8.

To disclose the degradation process of meat, we used $A_{u v}$ to reflect the spectral changes. The spatial distribu- tion of $A_{U V}$ at different times (i-vi: $1-6 h$ ) using the initial $(t=0 h)$ spectra at each pixel as references are presented in Figure 9(a). The most distinctive changes occur in the red meat region. The histograms of $A_{U V}$ are presented in Figure 9(b), showing that the histogram features at $A_{U V}=0.14,0.19$ and 0.23 change with degradation time.

We further measured HSI hypercubes of pork in a controlled environment, by increasing/depleting oxygen in the environment to adjust the equilibrium concentrations of deoxy and oxygenated $\mathrm{Mb}$, and using post-mortem bacteria metabolism in meat to vary the concentration of MetMb. The resulting HSI data were subject to PCA. The first three PCA components, which are attributable to DeoxyMb, OxyMb and MetMb, ${ }^{14}$ are presented in Figure 10(a). We used these three eigen-spectra as a basis to deduce the relative concentrations of DeoxyMb, OxyMb and MetMb with HSI spectra measured at different time points. The result is summarised in Figure 10(b), which reveals that the relative concentration of DeoxyMb rapidly decreases from $55 \%$ to $5 \%$ after 3 -h degradation, whereas the relative concentrations of OxyMb increases from $45 \%$ to $70 \%$ in the first hour and then decreases steadily to $40 \%$ after 6-h degradation. On the other hand, MetMb steadily increases from $0 \%$ to more than $60 \%$ in 6 -h of degradation, accompanied by a monotonic decrease of $\mathrm{pH}$ from 6.8 to 6.5. The degradation chemistry of pork can be understood as follows: the DeoxyMb in red meat will first be converted to OxyMb by oxygenation. After 1-h degradation, both DeoxyMb and OxyMb are irreversibly converted to MetMb by bacteria metabolism, resulting in an accumulation of MetMb and acidification of the meat.

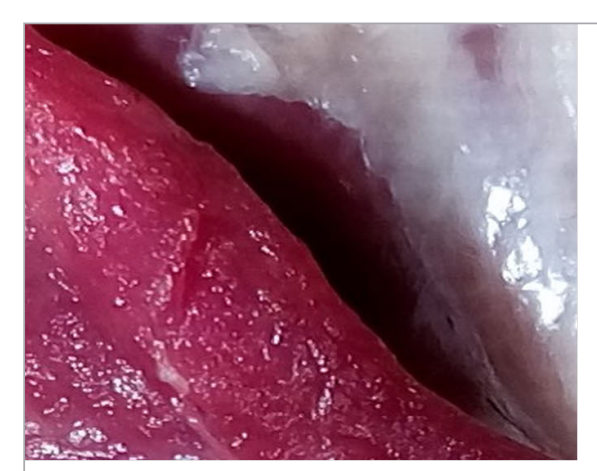

(a)

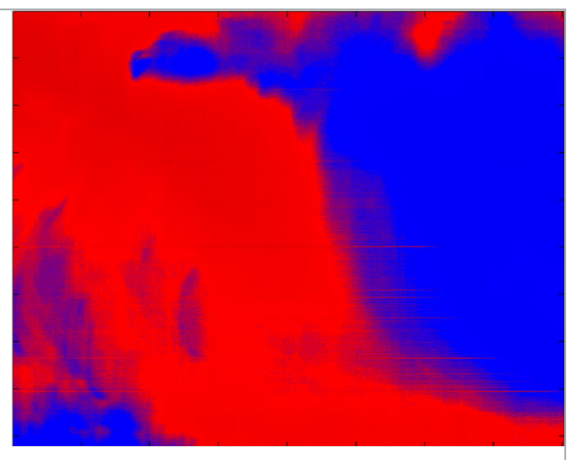

(b)

Figure 8. PCA of HSI data from pork at $t=0 \mathrm{~min}$. (a) Digital RGB colour image, (b) spatial distribution of the first two PCA components (highlight with red and blue). 


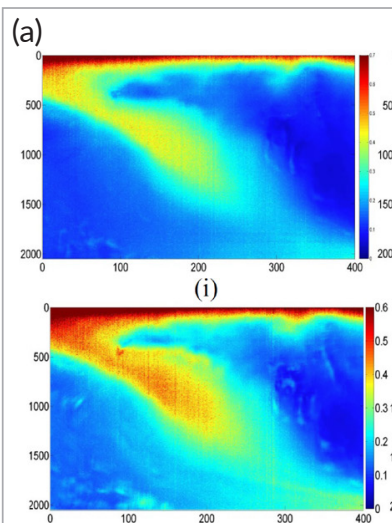

(iv)

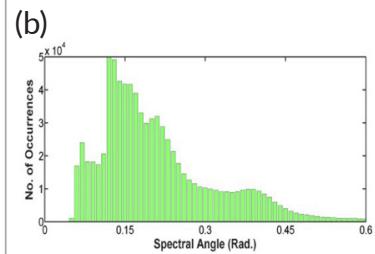

(i)

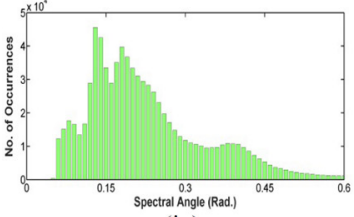

(iv)

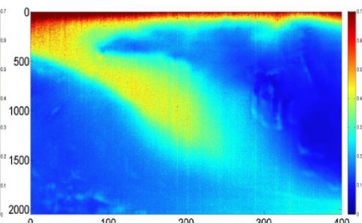

(ii)

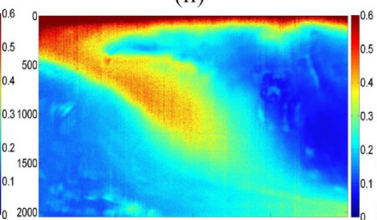

(v)

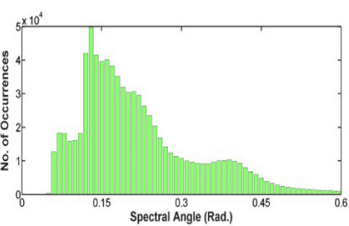

(ii)

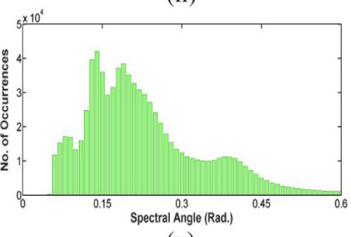

(v)



(iii)

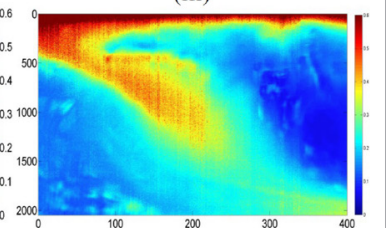

(vi)

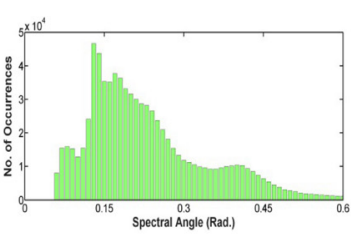

(111)

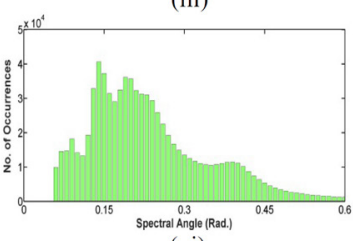

(vi)

Figure 9. Time series HSI data. (a) Spatial distribution of $A_{u v}$ using initial $(t=0 \mathrm{~h})$ pixel-wise spectra as references, and $(b)$ the corresponding histograms of $A_{U V}$ with (i)-(vi): 0-6h.
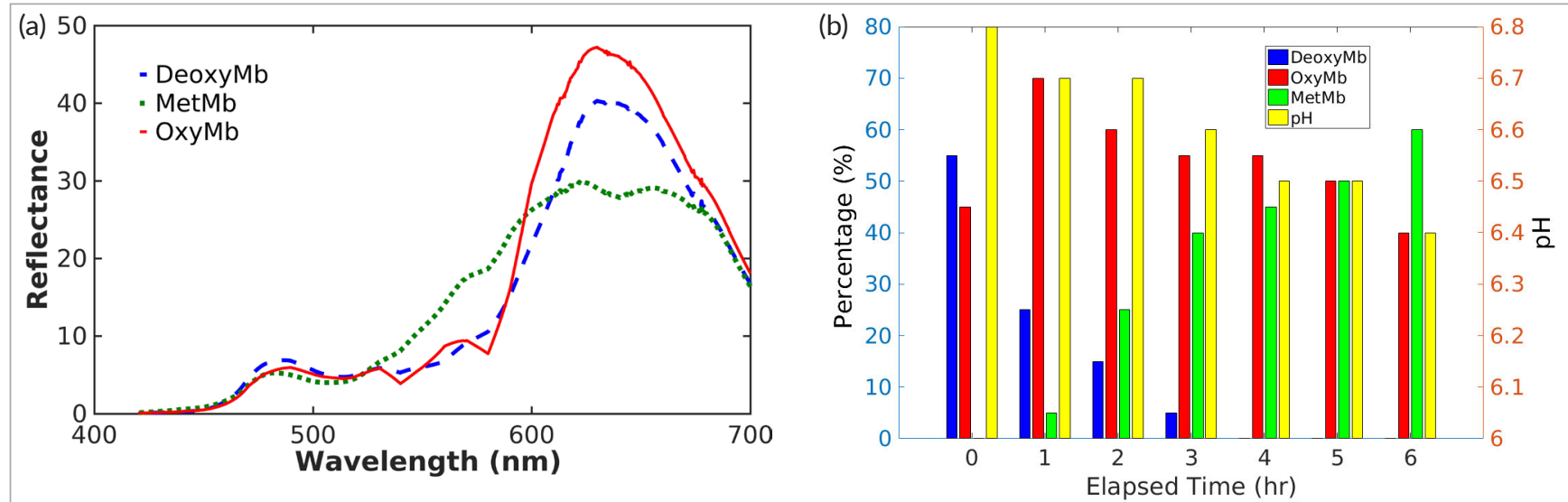

Figure 10. (a) The eigen-spectra of PCA deduced from HSI data measured under various conditions. (b) Relative percentages of HSI classes [DeoxyMb (blue), OxyMb (red) and MetMb (green)] and pH value (yellow) of meat are shown as a function of elapsed time.

\section{Conclusions}

In summary, we developed a hyperspectral imaging instrument based on telecentric optics with an internal scene-scan scheme and a long focal depth. Our HSI apparatus can capture a scene without moving the instrument or the tested object and yields an imaging resolution of $0.4 \mathrm{~mm}$ in a $10 \times 10 \mathrm{~cm}$ FoV and a spectral resolution of $1.3 \mathrm{~nm}$ in the $400-800 \mathrm{~nm}$ visible light region. This $\mathrm{HSI}$ apparatus can be built at a cost 
$<$ US $\$ 10 \mathrm{~K}$. We applied the technique to analyse the degradation chemistry of apple and pork. Based on the acquired $\mathrm{HSI}$ data, the degradation of apple can be classified into peels, flesh + peel, flesh and nucleus. This finding correlates well with the antioxidant activity in the four parts of an apple. We also succeeded in applying the HSI apparatus to reveal that DeoxyMb in red meat first converts to OxyMb, followed by a slow irreversible conversion to MetMb by bacteria metabolism, and results in an accumulation of MetMb and acidification of the meat. Our study indicates that the HSI instrument design and data analysis have the potential to perform direct identification of different ingredients and their spatial distribution in food products for objective quality evaluation.

\section{Acknowledgements}

This research was funded by the Ministry of Science and Technology of the Republic of China (grant number MOST 106-2112-M-009-019-MY3).

\section{References}

1. I.V. Meglinski and S.J. Matcher, "Quantitative assessment of skin layers absorption and skin reflectance spectra simulation in the visible and near-infrared spectral regions", Physiol Meas. 23, 741 (2002). doi: https://doi.org/10.1088/0967-3334/23/4/312

2. G. Lu and B. Fei, "Medical hyperspectral imaging: a review", J. Biomed Opt. 19, 010901 (2014). doi: https://doi.org/10.1117/1.JBO.19.1.010901

3. M. Joel and V. Tuan, "Optical properties of tissue", Chapter 2 in Biomedical Photonics Handbook. CRC Press, Boca Raton, FL, pp. 1-76 (2003).

4. D.N. Wadduwage, V.R. Singh, H. Choi, Z. Yaqoob, H. Heemskerk, P. Matsudaira and P.T.C. So, "Nearcommon-path interferometer for imaging Fouriertransform spectroscopy in wide-field microscopy", Optica 4, 546 (2017). doi: https://doi.org/10.1364/ OPTICA.4.000546

5. Q. Li, X. He, Y. Wang, H. Liu, D. Xu and F. Guo, "Review of spectral imaging technology in biomedical engineering: achievements and challenges", J. Biomed. Opt. 18, 100901 (2013). doi: https://doi. org/10.1117/1.JBO.18.10.100901
6. N. Hagen and M.W. Kudenov, "Review of snapshot spectral imaging technologies", Optical Eng. 52, 090901 (2013). doi: https://doi.org/10.1117/1. OE.52.9.090901

7. S. Jin, W. Hui, Y. Wang, K. Huang, Q. Shi, C. Ying, D. Liu, Q. Ye, W. Zhou and J. Tian, "Hyperspectral imaging using the single-pixel Fourier transform technique", Sci. Rep. 7, 45209 (2017). doi: https://doi. org/10.1038/srep45209

8. I. August, Y. Oiknine, M. AbuLeil, I. Abdulhalim and A. Stern, "Miniature compressive ultra-spectral imaging system utilizing a single liquid crystal phase retarder", Sci. Rep. 6, 23524 (2016). doi: https://doi. org/10.1038/srep23524

9. R. Shogenji, Y. Kitamura, K. Yamada, S. Miyatake and J. Tanida, "Multispectral imaging using compact compound optics", Optics Express 12, 1643 (2004). doi: https://doi.org/10.1364/OPEX.12.001643

10. P. Wang and R. Menon, "Computational multispectral video imaging", J. Opt. Soc. Amer. A 35, 189 (2018). doi: https://doi.org/10.1364/ JOSAA.35.000189

11. S.-H. Baek, I. Kim, D. Gutierrez and M. H. Kim, "Compact single-shot hyperspectral imaging using a prism", ACM Trans. Graphics 36(6), 217 (2017). doi: https://doi.org/10.1145/3130800.3130896

12. J. Qin, M.S. Kim, K. Chao, D.E. Chan, S.R. Delwiche and B.-K. Cho, Appl. Sci. 7(2), 125 (2017). doi: https://doi.org/10.3390/app7020125

13. M. Laikin, Lens Design, $3^{\text {rd }}$ Edn. CRC Press, Boca Raton, FL, pp. 233-265 (2001).

14. M. Hunt and A. King, Meat Color Measurement Guidelines, $1^{\text {st }}$ Edn. American Meat Science Association, Champaign, IL, Vol. 1 (2012).

15. G. Elmasry, D.F. Barbin, D.-W. Sun and P. Allen, "Meat quality evaluation by hyperspectral imaging technique: an overview", Crit. Rev. Food Sci. Nutr. 52, 689 (2012). doi: https://doi.org/10.1080/10408398. 2010.507908

16. D. Barbin, G. Elmasry, D.-W. Sun and P. Allen, "Nearinfrared hyperspectral imaging for grading and classification of pork", Meat Sci. 90, 259 (2012). doi: https://doi.org/10.1016/j.meatsci.2011.07.011

17. D. Barbin, G. Elmasry, D.-W. Sun and P. Allen, "Nondestructive determination of chemical composition in intact and minced pork using near-infrared hyperspectral imaging", Food Chem. 138, 1162 (2013). doi: https://doi.org/10.1016/j.foodchem.2012.11.120 
18. D.-W. Sun, Hyperspectral Imaging for Food Quality Analysis and Control. Elsevier (2010). ISBN 978-0-12374753-2

19. K. Wolfe, X.Z. Wu and R.H. Liu, "Antioxidant activity of apple peels", J. Agric. Food Chem. 51, 609 (2003). doi: https://doi.org/10.1021/jf020782a

20. R. Yoruk and M. R. Marshall, "Physicochemical properties and function of plant polyphenol oxidase: a review", J. Food Biochem. 27, 361 (2003). doi: https:// doi.org/10.1111/j.1745-4514.2003.tb00289.x

21. Labsphere, https://www.labsphere.com/site/assets/ files/2553/a-guide-to-reflectance-materials-andcoatings.pdf.

22. D. Manolakis, D. Marden and G.A. Shaw, "Hyperspectral image processing for automatic target detection applications", Lincoln Lab. J. 14, 79 (2003).

23. P.-H. Hsu, "Feature extraction of hyperspectral images using wavelet and matching pursuit", ISPRS J. Photogramm. Remote Sens. 62, 78 (2007). doi: https:// doi.org/10.1016/j.isprsjprs.2006.12.004

24. P. Ghamisi, M.S. Couceiro and J.A. Benediktsson, "A novel feature selection approach based on FODPSO and SVM", IEEE Trans. Geos. Remote Sens.

53, 2935 (2015). doi: https://doi.org/10.1109/ TGRS.2014.2367010

25. M. Fauvel, J. Benediktsson, J. Chanussot and J. Sveinsson, "Spectral and spatial classification of hyperspectral data using SVMs and morphological profiles", IEEE Trans. Geos. and Remote Sens.

46, 3804 (2008). doi: https://doi.org/10.1109/ TGRS.2008.922034

26. Y. Bazi and F. Melgani, "Toward an optimal SVM classification system for hyperspectral remote sensing images", IEEE Trans. Geos. and Remote Sens. 44, 3374 (2006). doi: https://doi.org/10.1109/ TGRS.2006.880628

27. J.M. Amigo, H. Babamoradi and S. Elcoroaristizabal, "Hyperspectral image analysis. A tutorial", Anal. Chim.
Acta 896, 34 (2015). doi: https://doi.org/10.1016/j. aca.2015.09.030

28. S. Matteoli, M. Diani and G. Corsini, "A tutorial overview of anomaly detection in hyperspectral images", IEEE Aero. Electron. Syst. Mag. 25, 5 (2010). doi: https://doi.org/10.1109/MAES.2010.5546306

29. R. Dorrepaal, C. Malegori and A. Gowen, "Tutorial: time series hyperspectral image analysis", J. Near Infrared Spectrosc. 24, 89 (2016). doi: https://doi. org/10.1255/jnirs.1208

30. A. Bjorgan and L.L. Randeberg, "Real-time noise removal for line-scanning hyperspectral devices using a minimum noise fraction-based approach", Sensors 15, 3362 (2015). doi: https://doi. org/10.3390/s150203362

31. I. Jolliffe, Springer Series in Statistics: Principal Component Analysis. Springer Nature (2002).

32. S. Berisha, S. Chang, S. Saki, D. Daeinejad, Z. He, R. Mankar and D. Mayerich, "SIproc: an open-source biomedical data processing platform for large hyperspectral images", Analyst 142, 1350 (2017). doi: https://doi.org/10.1039/C6ANO2082H

33. C. Shi, Y. Dai, X. Xu, Y. Xie, B. Xia and Q. Li, "UV/vis spectra studies on the polyphenol oxidase II (PPO II) from tobacco", Spectrosc. Lett. 6, 675 (2001). doi: https://doi.org/10.1081/SL-100107890

34. R. Pike, G. Lu, D. Wang, Z.G. Chen and B. Fei, "A minimum spanning forest-based method for noninvasive cancer detection with hyperspectral imaging", IEEE Trans. Biomed. Eng. 63, 653663 (2016). doi: https://doi.org/10.1109/TBME.2015.2468578

35. H. Li, Y. Ma, K. Liang and Y. Yu, "Rapid matching algorithm for hyperspectral image based on norm sifting", Chinese Opt. Lett. 10, 013001 (2012). doi: https://doi.org/10.3788/COL201210.013001 